\title{
Systematic study of the effect of short range correlations on the form factors and densities of $\mathrm{s}-\mathrm{p}$ and $\mathrm{s}-\mathrm{d}$ shell nuclei
}

\author{
S.E. Massen, H.C. Moustakidis \\ Department of Theoretical Physics, Aristotle University of Thessaloniki \\ GR-54006 Thessaloniki, Greece
}

September 26, 2018

\begin{abstract}
Analytical expressions of the one- and two-body terms in the cluster expansion of the charge form factors and densities of the $\mathrm{s}-\mathrm{p}$ and $\mathrm{s}-\mathrm{d}$ shell nuclei with $\mathrm{N}=\mathrm{Z}$ are derived. They depend on the harmonic oscillator parameter $b$ and the parameter $\beta$ which originates from the Jastrow correlation function. These expressions are used for the systematic study of the effect of short range correlations on the form factors and densities and of the mass dependence of the parameters $b$ and $\beta$. These parameters have been determined by fit to the experimental charge form factors. The inclusion of the correlations reproduces the experimental charge form factors at the high momentum transfers $\left(q \geq 2 \mathrm{fm}^{-1}\right)$. It is found that while the parameter $\beta$ is almost constant for the closed shell nuclei, ${ }^{4} \mathrm{He}$, ${ }^{16} \mathrm{O}$ and ${ }^{40} \mathrm{Ca}$, its values are larger (less correlated systems) for the open shell nuclei, indicating a shell effect in the closed shell nuclei.
\end{abstract}

PACS numbers: 21.10.Ft, 25.30.Bf, 21.45.+v, 21.60.Cs

\section{INTRODUCTION}

The calculation of the charge form factors, $F_{c h}(q)$, and density distributions, $\rho_{c h}(r)$ of nuclei, is a challenging and appealing problem [1]. A possibility to face this problem is by means of an independent particle model. This approach, which is particularly attractive because of its simplicity, fails to reproduce the high momentum transfer data from electron scattering in nuclei [2, 3, 4, 5, 6, 7, 8, 9, 10, 11]. For this reason a modification of the single particle (SP) potentials has to be suitably made.

In fact a short range repulsion in this potential seems advisable for light nuclei [12]. For example, with an harmonic oscillator $(\mathrm{HO})$ potential having in addition an infinite soft core, the $F_{c h}(q)$ of ${ }^{4} \mathrm{He}$ can be well reproduced, but for the heavier nuclei, such as ${ }^{12} \mathrm{C}$ and ${ }^{16} \mathrm{O}$, state dependent potentials seem necessary and even then the fit is not so good for higher q-values 
[12]. Another way is the introduction of a modified shell model with fractional occupation numbers for the various states [13, 14, 15, 16].

An approach, which is rather similar, is the introduction of the short range correlations (SRC) in the Slater determinant. Many attempts have been made in this direction, concerning mainly light closed shell nuclei in the framework of the Born-approximation [⿶, 5, 6, 0, 8, 9, 10, 11, 17, 18, 19].

Czyz and Lesniak [2] first showed that the diffraction character of the ${ }^{4} \mathrm{He}$ form factor can be qualitatively explained by means of Jastrow-type [20] correlations. Khana [3], using IwamotoYamada cluster expansion and retaining only one- and two-body cluster terms, showed that the inclusion of short range nucleon-nucleon correlations provide an adequate description for the known data on the elastic scattering of electrons by ${ }^{40} \mathrm{Ca}$ and make predictions for the behavior of the cross section at large momentum transfers. Ciofi Degli Atti, using the "single pair approximation" [4] and the Iwamoto-Yamada cluster expansion [5] in s-p shell nuclei, showed that the elastic electron scattering at high momentum transfers seems to give a strong indication of the presence of SRC in nuclei. Bohigas and Stringari [8] and Dal Ri et al [9] evaluated the effect of SRC on the one- and two-body densities by developing a low order approximation (LOA) in the framework of the Jastrow formalism. They showed that one-body quantities like the form factor provide an adequate test for the presence of SRC in nuclei, which indicates that the independent-particle wave functions cannot reproduce simultaneously the form factor and the momentum distribution of a correlated system. Stoitsov et al [11] generalized the model of Jastrow correlations, suggested by Bohigas and Stringari [8] within the LOA of Ref. [7], to heavier nuclei like ${ }^{16} \mathrm{O},{ }^{36} \mathrm{Ar}$ and ${ }^{40} \mathrm{Ca}$ reproducing very well the experimental data.

In the above approaches, different types of expansions were used. The expansions were connected with the number of the simultaneously correlated nucleons, and the order of the Jastrow correlation function, $f\left(r_{i j}\right)$, which were retained in the cluster expansion. Usually they are truncated up to the two-body terms which give significant contribution to various expectation values.

In a series of papers [17, 18, 19] an expression of the elastic charge form factor, $F_{c h}(q)$, truncated at the two-body term, was derived using the factor cluster expansion of Clark et al [21, 22, 23. This expression, which is a sum of one-body and two-body terms, and depends on the HO parameter and the correlation parameter through a Jastrow type correlation function, is used for the calculation of $F_{c h}(q)$ of ${ }^{4} \mathrm{He},{ }^{16} \mathrm{O}$ and ${ }^{40} \mathrm{Ca}$ and in an approximate way for the open $\mathrm{s}-\mathrm{p}$ and $\mathrm{s}-\mathrm{d}$ shell nuclei.

The motivation of the present work is the systematic study of the effect of SRC on the s-p and s-d shell nuclei by completely avoiding the approximation made in earlier work [17, 18, 19] for open shell nuclei, that is the expansion of the two-body terms in powers of the correlation parameter, in which only the leading terms had been retained. General expressions for the $F_{c h}(q)$ and $\rho_{c h}(r)$ were found using the factor cluster expansion of Clark et al and Jastrow correlation functions which introduce SRC. These expressions are functionals of the SP wave functions and not of the wave functions of the relative motion of two nucleons as was the case in many previous works $\llbracket, 10$, 17]. Because of that, it is easy to extrapolate them to the case of open shell nuclei and use them either for analytical calculations with HO wave functions or for numerical calculations when more realistic SP wave functions are used. An advantage of the present method is that the mass dependence of the HO parameter $b$ (with the presence of correlations) and the correlation parameter $\beta$ can be studied. These parameters have been determined, for the various $\mathrm{s}-\mathrm{p}$ and $\mathrm{s}-\mathrm{d}$ shell nuclei by fit of the theoretical $F_{c h}(q)$ to the 
experimental ones. It is found that while the parameter $\beta$ is almost constant for the closed shell nuclei, ${ }^{4} \mathrm{He},{ }^{16} \mathrm{O}$ and ${ }^{40} \mathrm{Ca}$, it takes larger values (less correlated systems) in the open shell nuclei, indicating a shell effect for the closed shells. The present method has also been used with wave functions of Skyrme-type interactions. In this case there is only one free parameter, the correlation parameter $\beta$ and the two body-term is a small correction to the one body-term of the density.

The paper is organized as follows. In Section II, the general expressions of the correlated form factors and density distributions are derived using a Jastrow correlation function. In section III the analytical expressions of the above quantities for the $\mathrm{s}-\mathrm{p}$ and $\mathrm{s}-\mathrm{d}$ shell nuclei, in the case of the HO orbitals, are given. Numerical results are reported and discussed in section $\mathrm{IV}$, while an outline of the present work is given in section $\mathrm{V}$.

\section{CORRELATED DENSITY DISTRIBUTIONS AND FORM FACTORS}

If we denote the model operator, which introduces SRC, by $\mathcal{F}$, an eigenstate $\Phi$ of the model system corresponds to an eigenstate

$$
\Psi=\mathcal{F} \Phi
$$

of the true system.

Several restrictions can be made on the model operator $\mathcal{F}$, as for example, that it depends on (the spins, isospins and) relative co-ordinates and momenta of the particles in the system, it is a scalar with respect to rotations e.t.c. [24]. Further, it is required that $\mathcal{F}$ is translationally invariant and symmetrical in its argument $1 \cdots i \cdots A$ and possesses the cluster property. That is if any subset, $i_{1} \cdots i_{p}$, of the particles is removed far from the rest, $i_{p+1} \cdots i_{A}, \mathcal{F}$ decomposes into a product of two factors, $\mathcal{F}(1 \cdots A)=\mathcal{F}\left(i_{1} \cdots i_{p}\right) \mathcal{F}\left(i_{p+1} \cdots i_{A}\right)$ [23]. In the present work $\mathcal{F}$ is taken to be of the Jastrow-type [20],

$$
\mathcal{F}=\prod_{i<j}^{A} f\left(r_{i j}\right)
$$

where $f\left(r_{i j}\right)$ is the state-independent correlation function of the form:

$$
f\left(r_{i j}\right)=1-\exp \left[-\beta\left(\mathbf{r}_{i}-\mathbf{r}_{j}\right)^{2}\right] .
$$

The correlation function $f\left(r_{i j}\right)$ goes to one for large values of $r_{i j}=\left|\mathbf{r}_{i}-\mathbf{r}_{j}\right|$ and it goes to zero for $r_{i j} \rightarrow 0$. It is obvious that the effect of SRC, introduced by the function $f\left(r_{i j}\right)$, becomes large when the SRC parameter $\beta$ becomes small and vice versa.

The charge form factor of a nucleus, in Born-approximation, can be written

$$
F_{c h}(q)=f_{p}(q) f_{D F}(q) f_{C M}(q) F_{p}(q)
$$

where $f_{p}(q)$ and $f_{D F}(q)$ are the correction for the finite proton size and the Darwin-Foldy relativistic correction, respectively [25], $f_{C M}(q)$ is the Tassie-Barker [26] center-of-mass correction and $F_{p}(q)$ the point form factor of the nucleus which is the expectation value of the one-body operator,

$$
\mathbf{O}_{q}=\sum_{i=1}^{A} \mathbf{o}_{q}(i)=\sum_{i=1}^{A} \exp \left[i \mathbf{q} \mathbf{r}_{i}\right]
$$


That is,

$$
F_{p}(q)=\frac{\left\langle\Psi\left|\mathbf{O}_{q}\right| \Psi\right\rangle}{\langle\Psi \mid \Psi\rangle}=N\left\langle\Psi\left|\mathbf{O}_{q}\right| \Psi\right\rangle=N\left\langle\mathbf{O}_{q}\right\rangle
$$

where $N=\left\langle\Psi\left(r_{1}, r_{2}, \cdots, r_{A}\right) \mid \Psi\left(r_{1}, r_{2}, \cdots, r_{A}\right)\right\rangle^{-1}$ is the normalization factor which is determined so that $F_{c h}(0)=F_{p}(0)=1$ or $4 \pi \int_{0}^{\infty} \rho(r) r^{2} \mathrm{~d} r=1$.

The point density distribution has the form

$$
\rho_{p}(r)=\frac{\left\langle\Psi\left|\mathbf{O}_{r}\right| \Psi\right\rangle}{\langle\Psi \mid \Psi\rangle}=N\left\langle\Psi\left|\mathbf{O}_{r}\right| \Psi\right\rangle=N\left\langle\mathbf{O}_{r}\right\rangle
$$

where

$$
\mathbf{O}_{r}=\sum_{i=1}^{A} \mathbf{o}_{r}(i)=\sum_{i=1}^{A} \delta\left(\mathbf{r}-\mathbf{r}_{i}\right)
$$

\subsection{EXPRESSIONS OF THE CORRELATED DENSITY DISTRI- BUTIONS AND FORM FACTORS}

In order to evaluate the point density distribution, $\rho_{p}(r)$, we consider, first, the generalized normalization integral,

$$
I(\alpha)=\left\langle\Psi\left|\exp \left[\alpha I(0) \mathbf{O}_{r}\right]\right| \Psi\right\rangle,
$$

corresponding to the operator $\mathbf{O}_{r}$, from which we have,

$$
\left\langle\mathbf{O}_{r}\right\rangle=\left[\frac{\partial \ln I(\alpha)}{\partial \alpha}\right]_{\alpha=0} .
$$

For the cluster analysis of equation (10), following the factor cluster expansion of Ristig and Clark [21, 22, 23], we consider the sum-product integrals, $I_{i}(\alpha), I_{i j}(\alpha), \cdots$, for the subsystems of the A-nucleon system and a factor cluster decomposition of these integrals. The expectation value of the density distribution operator is written in the form,

$$
\left\langle\mathbf{O}_{r}\right\rangle=\left\langle\mathbf{O}_{r}\right\rangle_{1}+\left\langle\mathbf{O}_{r}\right\rangle_{2}+\cdots+\left\langle\mathbf{O}_{r}\right\rangle_{A}
$$

where

$$
\begin{gathered}
\left\langle\mathbf{O}_{r}\right\rangle_{1}=\sum_{i=1}^{A}\left[\frac{\partial \ln I_{i}(\alpha)}{\partial \alpha}\right]_{\alpha=0}=\sum_{i=1}^{A}\left\langle i\left|\mathcal{F}_{1}^{\dagger} \mathbf{o}_{r}(1) \mathcal{F}_{1}\right| i\right\rangle \\
\left\langle\mathbf{O}_{r}\right\rangle_{2}=\sum_{i<j}^{A} \frac{\partial}{\partial \alpha}\left[\ln I_{i j}(\alpha)-\ln I_{i}(\alpha)-\ln I_{j}(\alpha)\right]_{\alpha=0} \\
=\sum_{i<j}^{A}\left\langle i j\left|\mathcal{F}_{12}^{\dagger}\left(\mathbf{o}_{r}(1)+\mathbf{o}_{r}(2)\right) \mathcal{F}_{12}\right| i j\right\rangle_{a}-\sum_{i<j}^{A}\left[\left\langle i\left|\mathbf{o}_{r}(1)\right| i\right\rangle+\left\langle j\left|\mathbf{o}_{r}(2)\right| j\right\rangle\right]
\end{gathered}
$$

and so on. $\mathcal{F}_{1}$ is chosen to be the identity operator.

The cluster expansion establishes the separation of one-body, two-body, $\cdots$, A-body correlation effects on the density. Three- and many-body terms will be neglected in the present 
analysis. Thus, in the two-body approximation, writing also the two sums of equation (13) in a different form, $\rho_{p}(r)$ is written as follows,

$$
\rho_{p}(r)=N\left\langle\mathbf{O}_{r}\right\rangle \approx N\left[\left\langle\mathbf{O}_{r}\right\rangle_{1}+\left\langle\mathbf{O}_{r}\right\rangle_{22}-\left\langle\mathbf{O}_{r}\right\rangle_{21}\right]
$$

where

$$
\begin{aligned}
\left\langle\mathbf{O}_{r}\right\rangle_{1} & =\sum_{i=1}^{A}\left\langle i\left|\mathbf{o}_{r}(1)\right| i\right\rangle \\
\left\langle\mathbf{O}_{r}\right\rangle_{22} & =2 \sum_{i<j}^{A}\left\langle i j\left|\mathcal{F}_{12}^{\dagger} \mathbf{O}_{r}(1) \mathcal{F}_{12}\right| i j\right\rangle_{a} \\
\left\langle\mathbf{O}_{r}\right\rangle_{21} & =2 \sum_{i<j}^{A}\left\langle i j\left|\mathbf{O}_{r}(1)\right| i j\right\rangle_{a} .
\end{aligned}
$$

If the two-body operator is taken to be the correlation function given by (3), then

$$
\mathcal{F}_{12}^{\dagger} \mathcal{F}_{12}=1-2 g\left(r_{1}, r_{2}, \beta\right)+g\left(r_{1}, r_{2}, 2 \beta\right)
$$

where

$$
g\left(r_{1}, r_{2}, z\right)=\exp \left[-z r_{1}^{2}\right] \exp \left[-z r_{2}^{2}\right] \exp \left[2 z r_{1} r_{2} \cos \omega_{12}\right], \quad z=\beta, 2 \beta,
$$

and the term $\left\langle\mathbf{O}_{r}\right\rangle_{22}$ is written,

$$
\left\langle\mathbf{O}_{r}\right\rangle_{22}=\left\langle\mathbf{O}_{r}\right\rangle_{21}-2 O_{22}(r, \beta)+O_{22}(r, 2 \beta)
$$

where

$$
O_{22}(r, z)=2 \sum_{i<j}^{A}\left\langle i j\left|\mathbf{o}_{r}(1) g\left(r_{1}, r_{2}, z\right)\right| i j\right\rangle_{a} .
$$

So, $\rho_{p}(r)$ takes the form,

$$
\rho_{p}(r) \approx N\left[\left\langle\mathbf{O}_{r}\right\rangle_{1}-2 O_{22}(r, \beta)+O_{22}(r, 2 \beta)\right] .
$$

The terms $\left\langle\mathbf{O}_{r}\right\rangle_{1}$ and $O_{22}(r, z)$ and the density $\rho_{p}(r)$ can be expressed also in the convenient form:

$$
\begin{gathered}
\left\langle\mathbf{O}_{r}\right\rangle_{1}=\rho_{S D}(\mathbf{r}), \\
O_{22}(r, z)=\int g\left(r, r_{2}, z\right)\left[\rho_{S D}(\mathbf{r}) \rho_{S D}\left(\mathbf{r}_{2}\right)-\rho_{S D}^{2}\left(\mathbf{r}, \mathbf{r}_{2}\right)\right] \mathrm{d} \mathbf{r}_{2}, \\
\rho_{p}(r) \approx N\left[\rho_{S D}(\mathbf{r})+\int\left[g\left(r, r_{2}, 2 \beta\right)-2 g\left(r, r_{2}, \beta\right)\right]\left[\rho_{S D}(\mathbf{r}) \rho_{S D}\left(\mathbf{r}_{2}\right)-\rho_{S D}^{2}\left(\mathbf{r}, \mathbf{r}_{2}\right)\right]\right] \mathrm{d} \mathbf{r}_{2},
\end{gathered}
$$

where $\rho_{S D}\left(\mathbf{r}_{1}, \mathbf{r}_{2}\right)$ is the uncorrelated density matrix associated with the Slater determinant,

$$
\rho_{S D}\left(\mathbf{r}_{1}, \mathbf{r}_{2}\right)=\sum_{i=1}^{A} \phi_{i}^{*}\left(\mathbf{r}_{1}\right) \phi_{i}\left(\mathbf{r}_{2}\right)
$$

The diagonal elements of this gives the one body density distribution,

$$
\rho_{S D}\left(\mathbf{r}_{1}\right)=\rho_{S D}\left(\mathbf{r}_{1}, \mathbf{r}_{1}\right) .
$$


It should be noted that, a similar expression for $\rho_{p}(r)$, given by equation (25), was derived by Gaudin et al. [7] in the framework of LOA. In LOA the Jastrow wave function, $\Psi$, of the nucleus was expanded in terms of the functions: $\tilde{g}=f^{2}\left(r_{i j}\right)-1$ and $h=f\left(r_{i j}\right)-1$ and was truncated up to the second order of $h$ and the first order of $\tilde{g}$. This expansion contains one- and two-body terms and a part of the three-body term which was chosen so that the normalization of the wave function was preserved. Expression (25) of the present work has only one- and two-body terms and the normalization of the wave function is preserved by the normalization factor $N$.

In the above expression of $\rho_{p}(r)$, the one-body contribution to the density is well known and given by the equation,

$$
\left\langle\mathbf{O}_{r}\right\rangle_{1}=4 \sum_{n l} \eta_{n l}(2 l+1) \frac{1}{4 \pi} \phi_{n l}^{*}(r) \phi_{n l}(r)
$$

where $\eta_{n l}$ is the occupation probability of the state $n l$ ( 0 or 1 in the case of closed shell nuclei) and $\phi_{n l}(r)$ is the radial part of the SP wave function.

An expression for the two-body term is usually found by making a transformation to the relative and the center-of-mass coordinates of the two interacting nucleons [1, 10, 17]. This is because the Jastrow function $f\left(r_{i j}\right)$ depends on the relative coordinates of the two nucleons. Here, the expression for the two-body term, that is of the term $O_{22}(r, z)$, will be found by expanding the factor $\exp \left[2 z r_{1} r_{2} \cos \omega_{12}\right]$ in spherical harmonics [27, 28]. That is

$$
\exp \left[2 z r_{1} r_{2} \cos \omega_{12}\right]=2 \pi \sum_{k m_{k}} U_{k}\left(2 z r_{1} r_{2}\right) Y_{k m_{k}}^{*}\left(\Omega_{1}\right) Y_{k m_{k}}\left(\Omega_{2}\right)
$$

where

$$
\begin{aligned}
U_{k}\left(2 z r_{1} r_{2}\right) & =\int_{-1}^{1} \exp \left(2 z r_{1} r_{2} \cos \omega_{12}\right) P_{k}\left(\cos \omega_{12}\right) \mathrm{d}\left(\cos \omega_{12}\right) \\
& =2 i_{k}\left(2 z r_{1} r_{2}\right)
\end{aligned}
$$

$i_{k}(x)$ is the modified spherical Bessel function.

Using the algebra of spherical harmonics, the term: $O_{22}(r, z)$ takes the form:

$$
\begin{aligned}
O_{22}(r, z)= & 4 \sum_{n_{i} l_{i}, n_{j} l_{j}} \eta_{n_{i} l_{i}} \eta_{n_{j} l_{j}}\left(2 l_{i}+1\right)\left(2 l_{j}+1\right) \times \\
& {\left[4 A_{n_{i} l_{i} n_{j} l_{j}}^{n_{i} l_{i} n_{j} l_{j} k}(r, z)-\sum_{k=0}^{l_{i}+l_{j}}\left\langle l_{i} 0 l_{j} 0 \mid k 0\right\rangle^{2} A_{n_{i} l_{i} n_{j} l_{j}}^{n_{j} l_{j} n_{i} l_{i} k}(r, z)\right], \quad z=\beta, 2 \beta, }
\end{aligned}
$$

where

$$
\begin{aligned}
A_{n_{1} l_{1} n_{2} l_{2}}^{n_{3} l_{3} n_{4} l_{4} k}(r, z)= & \frac{1}{4 \pi} \phi_{n_{1} l_{1}}^{*}(r) \phi_{n_{3} l_{3}}(r) \exp \left[-z r^{2}\right] \times \\
& \int_{0}^{\infty} \phi_{n_{2} l_{2}}^{*}\left(r_{2}\right) \phi_{n_{4} l_{4}}\left(r_{2}\right) \exp \left[-z r_{2}^{2}\right] i_{k}\left(2 z r r_{2}\right) r_{2}^{2} \mathrm{~d} r_{2}
\end{aligned}
$$

Thus the expression of the term $O_{22}(r, z)$ depends on the SP wave functions and so it is suitable to be used either for analytical calculations with the $\mathrm{HO}$ potential or for numerical 
calculations with more realistic SP potentials. Expressions (28) and (31) were derived for the closed shell nuclei with $\mathrm{N}=\mathrm{Z}$, where $\eta_{n l}$ is 0 or 1 . For the open shell nuclei (with $\mathrm{N}=\mathrm{Z}$ ) we use the same expressions, where now: $0 \leq \eta_{n l} \leq 1$. In this way the mass dependence of the correlation parameter $\beta$ and the HO parameter $b$ can be studied.

Finally, using the known values of the Clebsch-Gordan coefficients and provided that $\phi_{n l}^{*}(r)=\phi_{n l}(r)$, i.e.: $A_{n_{1} l_{1} n_{2} l_{2}}^{n_{3} l_{3} n_{4} l_{4} k}(r, z)=A_{n_{3} l_{3} n_{4} l_{4} k}^{n_{1} l_{1} n_{2} l_{2}}(r, z)$, equation (31), for the case of s-p and $\mathrm{s}-\mathrm{d}$ shell nuclei, takes the form:

$$
\begin{aligned}
O_{22}(r, z)= & 4\left[3 A_{0000}^{0000}(r, z) \eta_{1 s}^{2}+\left(33 A_{0101}^{01010}(r, z)-6 A_{0101}^{01012}(r, z)\right) \eta_{1 p}^{2}+3 A_{1010}^{10100}(r, z) \eta_{2 s}^{2}+\right. \\
& \left(95 A_{0202}^{02020}(r, z)-\frac{50}{7} A_{0202}^{02022}(r, z)-\frac{90}{7} A_{0202}^{02024}(r, z)\right) \eta_{1 d}^{2}+ \\
& \left(12 A_{0001}^{00010}(r, z)+12 A_{0100}^{01000}(r, z)-6 A_{0001}^{01001}(r, z)\right) \eta_{1 s} \eta_{1 p}+ \\
& \left(20 A_{0002}^{00020}(r, z)+20 A_{0200}^{02000}(r, z)-10 A_{0002}^{02002}(r, z)\right) \eta_{1 s} \eta_{1 d}+ \\
& \left(60 A_{0102}^{01020}(r, z)+60 A_{0201}^{02010}(r, z)-12 A_{0102}^{02011}(r, z)-18 A_{0102}^{02013}(r, z)\right) \eta_{1 p} \eta_{1 d}+ \\
& \left(4 A_{0010}^{00100}(r, z)+4 A_{1000}^{10000}(r, z)-2 A_{0010}^{10000}(r, z)\right) \eta_{1 s} \eta_{2 s}+ \\
& \left(12 A_{0110}^{01100}(r, z)+12 A_{1001}^{10010}(r, z)-6 A_{0110}^{10011}(r, z)\right) \eta_{1 p} \eta_{2 s}+ \\
& \left.\left(20 A_{0210}^{02100}(r, z)+20 A_{1002}^{10020}(r, z)-10 A_{0210}^{10022}(r, z)\right) \eta_{1 d} \eta_{2 s}\right]
\end{aligned}
$$

The point form factor $F_{p}(q)$ can be derived in two equivalent ways. The first one is to follow the same cluster expansion as in the case of the density distribution and the second one is to take the Fourier transform of the density distribution $\rho_{p}(r)$,

$$
F_{p}(q)=\int \exp [i \mathbf{q} \mathbf{r}] \rho_{p}(r) \mathrm{d} \mathbf{r} .
$$

In both cases, the form factor takes the following form:

$$
F_{p}(q) \approx N\left[\left\langle\mathbf{O}_{q}\right\rangle_{1}-2 \tilde{O}_{22}(q, \beta)+\tilde{O}_{22}(q, 2 \beta)\right] .
$$

In the above expression, the one-body term is given by the equation

$$
\left\langle\mathbf{O}_{q}\right\rangle_{1}=4 \sum_{n l} \eta_{n l}(2 l+1) \int_{0}^{\infty} \phi_{n l}^{*}(r) \phi_{n l}(r) j_{0}(q r) r^{2} d r
$$

while the two-body term $\tilde{O}_{22}(q, z)$ is given by the right hand side of equations (31) and (33) by replacing the matrix elements $A_{n_{1} l_{1} n_{2} l_{2}}^{n_{3} l_{3} n_{4} l_{4} k}(r, z)$ by $\tilde{A}_{n_{1} l_{1} n_{2} l_{2}}^{n_{3} l_{3} n_{4} l_{4} k}(q, z)$ given by the equation,

$$
\begin{aligned}
\tilde{A}_{n_{1} l_{1} n_{2} l_{2}}^{n_{3} l_{3} n_{4} l_{4} k}(q, z)= & \int_{0}^{\infty} \phi_{n_{1} l_{1}}^{*}\left(r_{1}\right) \phi_{n_{3} l_{3}}\left(r_{1}\right) \exp \left[-z r_{1}^{2}\right] j_{0}\left(q r_{1}\right) r_{1}^{2} \mathrm{~d} r_{1} \times \\
& \int_{0}^{\infty} \phi_{n_{2} l_{2}}^{*}\left(r_{2}\right) \phi_{n_{4} l_{4}}\left(r_{2}\right) \exp \left[-z r_{2}^{2}\right] i_{k}\left(2 z r_{1} r_{2}\right) r_{2}^{2} \mathrm{~d} r_{2},
\end{aligned}
$$

where $j_{0}(x)$ and $i_{k}(x)$ are the spherical Bessel and the modified spherical Bessel functions respectively. 


\section{$2.2 \quad l-s$ COUPLING SCHEME}

In the cases of mean field potentials with l-s coupling, the wave function of the $n l j$ state has the form:

$$
\psi_{n l j}\left(r_{1}\right)=\phi_{n l j}\left(r_{1}\right) \sum_{m_{l}, m_{s}}\left\langle l m_{l} s m_{s} \mid j m_{j}\right\rangle Y_{l}^{m_{l}}(\Omega) \chi_{s}^{m_{s}}(1) \chi_{t}^{m_{t}}(1),
$$

where $\phi_{n l j}\left(r_{1}\right)$ is the radial part of the wave function of the state $n l j$ and $\chi_{s}^{m_{s}}(1), \chi_{t}^{m_{t}}(1)$ are the spin and the isospin wave functions respectively.

Following the same procedure as in sub-section 2.1, the same expression (22) for $\rho_{p}(r)$ has been found. In this case the terms $\left\langle\mathbf{O}_{r}\right\rangle_{1}$ and $O_{22}(r, z)$ have the form:

$$
\left\langle\mathbf{O}_{r}\right\rangle_{1}=2 \sum_{n l} \eta_{n l j}(2 j+1) \frac{1}{4 \pi} \phi_{n l j}^{*}(r) \phi_{n l j}(r)
$$

and

$$
\begin{aligned}
O_{22}(r, z)= & 2 \sum_{n_{i} l_{i} j_{i}, n_{j} l_{j} j_{j}} \eta_{n_{i} l_{i} j_{i}} \eta_{n_{j} l_{j} j_{j}}\left(2 j_{i}+1\right)\left(2 j_{j}+1\right) \times \\
& {\left[2 A_{n_{i} l_{i} j_{i} n_{j} l_{j} j_{j}}^{n_{i} l_{i} j_{i} n_{j} l_{j} j_{j} k}(r, z)-\frac{1}{2} \sum_{k=0}^{l_{i}+l_{j}}\left\langle l_{i} 0 l_{j} 0 \mid k 0\right\rangle^{2} A_{n_{i} l_{i} j_{i} n_{j} l_{j} j_{j}}^{n_{j} l_{j} j_{j} n_{i} l_{i} j_{i} k}(r, z)\right], \quad z=\beta, 2 \beta,(}
\end{aligned}
$$

where the matrix elements $A_{n_{1} l_{1} j_{1} n_{2} l_{2} j_{2}}^{n_{3} l_{3} j_{3} n_{4} l_{4} j_{4} k}(r, z)$ are given again by equation (32) replacing the wave functions $\phi_{n l}(r)$ by the wave functions $\phi_{n l j}(r)$. Equation (40) can also be written in a similar form with that of equation (33) with the difference that there are now more terms. This expression goes to (33) if there is no l-s coupling.

In the evaluation of the density matrix $\rho_{S D}\left(\mathbf{r}_{1}, \mathbf{r}_{2}\right)$, which is necessary for the derivation of the expression (40) of the term $O_{22}(r, z)$, we made the following approximation: In the sum over the spin coordinates, only terms of pairs of particles having the same third spin component are taken into account. The contribution of the terms which contain pairs with opposite third spin component is small and is neglected [29, 30]. In this scheme the form factor is calculated numerically by Fourier transform of $\rho_{p}(r)$ employing equation (34).

\section{ANALYTICAL EXPRESSIONS}

In the case of the $\mathrm{HO}$ wave functions, with radial part,

$$
\phi_{n l}(r)=N_{n l} b^{-3 / 2}\left(\frac{r}{b}\right)^{l} L_{n}^{l+\frac{1}{2}}\left(\frac{r^{2}}{b^{2}}\right) \exp \left[-\frac{r^{2}}{2 b^{2}}\right], \quad N_{n l}=\left(\frac{2 n !}{\Gamma\left(n+l+\frac{3}{2}\right)}\right)^{1 / 2}
$$

where the Associated Laguerre polynomial is defined as in Ref. [31], analytical expressions of the one-body term and of the matrix elements $A_{n_{1} l_{1} n_{2} l_{2}}^{n_{3} l_{3} n_{4} l_{4} k}(r, z)$ and $\tilde{A}_{n_{1} l_{1} n_{2} l_{2}}^{n_{3} l_{3} n_{4} l_{4} k}(q, z)$, defined by equation (32) and (37), can be found. From these expressions, the analytical expressions of the terms $\mathrm{O}_{22}(r, z)$ and $\tilde{O}_{22}(q, z)$, defined by equation (33), can also be found.

The expression of the one-body term of the density and form factor has the form:

$$
\left\langle\mathbf{O}_{x}\right\rangle_{1}=C \mathrm{e}^{-\xi^{2}} \sum_{k=0}^{2} C_{2 k} \xi^{2 k}, \quad x=r, q
$$


where for the $\left\langle\mathbf{O}_{r}\right\rangle_{1}$ the variable $\xi$ and the coefficients $C$ and $C_{2 k}$ are:

$$
\begin{aligned}
\xi & =\frac{r}{b}, \quad C=\frac{2}{\pi^{3 / 2} b^{3}} \\
C_{0} & =2 \eta_{1 s}+3 \eta_{2 s} \\
C_{2} & =4\left(\eta_{1 p}-\eta_{2 s}\right) \\
C_{4} & =\frac{4}{3}\left(2 \eta_{1 d}+\eta_{2 s}\right)
\end{aligned}
$$

while for the $\left\langle\mathbf{O}_{q}\right\rangle_{1}$ the corresponding quantities are:

$$
\begin{aligned}
\xi & =\frac{1}{2} b q, \quad C=2 \\
C_{0} & =2\left(\eta_{1 s}+\eta_{2 s}+3 \eta_{1 p}+5 \eta_{1 d}\right) \\
C_{2} & =-\frac{4}{3}\left(3 \eta_{1 p}+10 \eta_{1 d}+2 \eta_{2 s}\right) \\
C_{4} & =\frac{4}{3}\left(2 \eta_{1 d}+\eta_{2 s}\right) .
\end{aligned}
$$

The analytical expression of the matrix element $A_{n_{1} l_{1} n_{2} l_{2}}^{n_{3} l_{3} n_{4} l_{4} k}(r, z)$, which are given by equation (32), has the form:

$$
\begin{aligned}
A_{n_{1} l_{1} n_{2} l_{2}}^{n_{3} l_{3} n_{4} l_{4} k}(r, z)= & \left(\prod_{i=1}^{4} N_{n_{i} l_{i}}\right) \frac{y^{k}}{16 \sqrt{\pi} b^{3}} \xi^{l_{1}+l_{3}+k} L_{n_{1}}^{l_{1}+\frac{1}{2}}\left(\xi^{2}\right) L_{n_{3}}^{l_{3}+\frac{1}{2}}\left(\xi^{2}\right) \exp \left[-\frac{1+2 y}{1+y} \xi^{2}\right] \times \\
& \sum_{w=0}^{n_{2}} \sum_{s=0}^{n_{4}} \frac{(-1)^{w+s}}{w ! s !}\left(\begin{array}{c}
n_{2}+l_{2}+\frac{1}{2} \\
n_{2}-w
\end{array}\right)\left(\begin{array}{c}
n_{4}+l_{4}+\frac{1}{2} \\
n_{4}-s
\end{array}\right) \times \\
& \frac{\left(\frac{1}{2}\left(l_{2}+l_{4}-k\right)+w+s\right) !}{(1+y)^{\frac{1}{2}\left(l_{2}+l_{4}+k+3\right) w+s}} L_{\frac{1}{2}\left(l_{2}+l_{4}-k\right)+w+s}^{k+\frac{1}{2}}\left(\frac{-y^{2}}{1+y} \xi^{2}\right)
\end{aligned}
$$

where $\xi=r / b$ and $y=z b^{2}(z=\beta, 2 \beta)$. It is mentioned that the Clebsch-Gordan coefficients $\left\langle l_{i} 0 l_{j} 0 \mid k 0\right\rangle$, which appear in equation (31) (where $k$ runs from 0 to $l_{i}+l_{j}$ ), are different from zero only when $l_{i}+l_{j}+k$ is an even integer and therefore $l_{2}+l_{4}-k$ is an even integer. Because of that the lower index of the Laguerre polynomial of equation (45) is an integer.

The above expression of $A_{n_{1} l_{1} n_{2} l_{2}}^{n_{3} l_{3} l_{4} k}(r, z)$ is of the form: $f\left(\xi^{2}\right) \exp \left[-\frac{1+2 y}{1+y} \xi^{2}\right]$, where $f\left(\xi^{2}\right)$ is a polynomial of $\xi^{2}$. The substitution of the expression of $A_{n_{1} l_{1} n_{2} l_{2}}^{n_{3} l_{3} n_{4} k}(r, z)$ to the expression of $\mathrm{O}_{22}(r, z)$, which is given by equation (33), leads to the analytical expression of the two-body term of the density. This expression, for the case of $\mathrm{s}-\mathrm{p}$ and $\mathrm{s}-\mathrm{d}$ shell nuclei, is again of the form $f\left(\xi^{2}\right) \exp \left[-\frac{1+2 y}{1+y} \xi^{2}\right]$ where now $f\left(\xi^{2}\right)$ is a polynomial of the form:

$$
f\left(\xi^{2}\right)=\sum_{k=0}^{4} C_{2 k}\left(\eta_{n_{k} l_{k}}, y\right) \xi^{2 k}
$$

The corresponding analytical expression of the matrix element $\tilde{A}_{n_{1} l_{1} n_{2} l_{2}}^{n_{3} l_{3} n_{4} l_{4} k}(q, z)$, which are given by equation (37), has the form:

$$
\tilde{A}_{n_{1} l_{1} n_{2} l_{2}}^{n_{3} l_{3} n_{4} l_{4} k}(q, z)=\frac{\pi}{16}\left(\prod_{i=1}^{4} N_{n_{i} l_{i}}\right) \exp \left[-\frac{1+y}{1+2 y} \xi^{2}\right] \sum_{p=0}^{n_{1}} \sum_{\mathrm{v}=0}^{n_{3}} \sum_{w=0}^{n_{2}} \sum_{s=0}^{n_{4}} \sum_{t=0}^{\frac{1}{2}\left(l_{2}+l_{4}-k\right)+w+s} \frac{(-1)^{p+\mathrm{v}+w+s+t}}{p ! \mathrm{v} ! w ! s ! t !} \times
$$




$$
\begin{aligned}
& \left(\begin{array}{c}
n_{1}+l_{1}+\frac{1}{2} \\
n_{1}-p
\end{array}\right)\left(\begin{array}{c}
n_{3}+l_{3}+\frac{1}{2} \\
n_{3}-\mathrm{v}
\end{array}\right)\left(\begin{array}{c}
n_{2}+l_{2}+\frac{1}{2} \\
n_{2}-w
\end{array}\right)\left(\begin{array}{c}
n_{4}+l_{4}+\frac{1}{2} \\
n_{4}-s
\end{array}\right) \times \\
& \frac{\Gamma\left(\frac{1}{2}\left(l_{2}+l_{4}+k+3\right)+w+s\right)\left(\frac{1}{2}\left(l_{1}+l_{3}+k\right)+p+\mathrm{v}+t\right) !\left(\frac{1}{2}\left(k-l_{2}-l_{4}\right)-w-s\right)_{t}}{\Gamma\left(t+k+\frac{3}{2}\right)} \times \\
& y^{2 t+k} \frac{(1+y)^{\frac{1}{2}\left(l_{1}+l_{3}-l_{2}-l_{4}\right)+p+\mathrm{v}-w-s}}{(1+2 y)^{\frac{1}{2}\left(l_{1}+l_{3}+k+3\right)+p+\mathrm{v}+t}} L_{\frac{1}{2}\left(l_{1}+l_{3}+k\right)+p+\mathrm{v}+t}^{\frac{1}{2}}\left(\frac{1+y}{1+2 y} \xi^{2}\right),
\end{aligned}
$$

where $\xi=q b / 2$ and $y=z b^{2}(z=\beta, 2 \beta)$. The lower index of the Laguerre polynomial of equation (47) is an integer because $l_{1}+l_{3}+k$ is an even integer.

The above expression of $\tilde{A}_{n_{1} l_{1} n_{2} l_{2}}^{n_{3} l_{3} l_{4} l_{4} k}(q, z)$ is of the form: $f\left(\xi^{2}\right) \exp \left[-\frac{1+y}{1+2 y} \xi^{2}\right]$, where $f\left(\xi^{2}\right)$ is a polynomial of $\xi^{2}$. The substitution of the expression of $\tilde{A}_{n_{1} l_{1} n_{2} l_{2}}^{n_{3} l_{3} n_{4} l_{4} k}(q, z)$ to the expression of $\tilde{O}_{22}(q, z)$, given by equation (33), leads to the analytical expression of the two-body term of the form factor. This expression, for the case of $\mathrm{s}-\mathrm{p}$ and $\mathrm{s}-\mathrm{d}$ shell nuclei, is again of the form $\tilde{f}\left(\xi^{2}\right) \exp \left[-\frac{1+y}{1+2 y} \xi^{2}\right]$ where now $\tilde{f}\left(\xi^{2}\right)$ is a polynomial similar to that of equation (46).

From the analytical expression of the form factor, the analytical expression of the mean square radius of a nucleus, which is the coefficient of $-q^{2} / 6$, can be found. This expression is of the form:

$$
\left\langle r^{2}\right\rangle=N\left[\left\langle r^{2}\right\rangle_{1}-2\left\langle r^{2}\left(\beta b^{2}\right)\right\rangle_{22}+\left\langle r^{2}\left(2 \beta b^{2}\right)\right\rangle_{22}\right]
$$

where

$$
\left\langle r^{2}\right\rangle_{1}=\left[6\left(\eta_{1 s}+\eta_{2 s}\right)+30 \eta_{1 p}+70 \eta_{1 d}\right] b^{2}
$$

and

$$
\left\langle r^{2}(y)\right\rangle_{22}=b^{2}(1+2 y)^{-13 / 2} \sum_{k=0}^{5} a_{k} y^{k}, \quad y=\beta b^{2}, 2 \beta b^{2} .
$$

The coefficients $a_{k}$ depend on the occupation probabilities of the various states. If we expand, the right hand side of equation (50), in powers of $1 / y\left(y=\beta b^{2}>1\right)$ and keep powers of $\beta b^{2}$ up to $\left(\beta b^{2}\right)^{-3 / 2}$, an approximate expression of the contribution of the two-body term to the radius takes the simple form:

$$
\left\langle r^{2}(y)\right\rangle_{22}=C b^{2}\left(\beta b^{2}\right)^{-3 / 2}
$$

where the coefficient $C$ depends on the occupation probabilities of the various states.

The analytical expressions of the form factor and the density, which were found previously, will be used in section IV for the fit of the theoretical charge form factors to the experimental ones and for the calculations of the charge density distributions for various $\mathrm{N}=\mathrm{Z}(\mathrm{s}-\mathrm{p}$ and $\mathrm{s}-\mathrm{d}$ shell) nuclei.

\section{RESULTS AND DISCUSSION}

The calculations of the charge form factors for various s-p and $\mathrm{s}-\mathrm{d}$ shell nuclei, with $\mathrm{N}=\mathrm{Z}$, have been carried out on the basis of equations (4) and (35) and the analytical expressions of the oneand two-body terms which were given in section III. Two cases have been examined, named Case 1 and Case 2, which correspond to the analytical calculations with HO wave functions 
without and with SRC respectively. In Case 1 there is one free parameter, the HO parameter $b$, while in Case 2 there are two free parameters, the parameter $b$ and the correlation parameter $\beta$. The parameters, in both cases, have been determined, for each nucleus separately, by least squares fit to the experimental $F_{c h}(q)$.

The best fit values of the parameters as well as of the values of $\chi^{2}$,

$$
\chi^{2}=\frac{1}{K} \sum_{i=1}^{K}\left[\left(F_{i}(t h e)-F_{i}(\exp )\right) / \Delta F_{i}(\exp )\right]^{2},
$$

are displayed in Table I. In the same table the calculated root mean square (RMS) charge radii $\left\langle r_{c h}^{2}\right\rangle^{1 / 2}$ and the contribution of the SRC to them,

$$
\left\langle r^{2}\right\rangle_{2}=\left\langle r_{c h}^{2}\right\rangle-\left\langle r_{c h}^{2}\right\rangle_{1} / A
$$

are displayed and compared with the corresponding experimental RMS radii. It is noted that $\left\langle r^{2}\right\rangle_{2}$ is independent from the centre-of-mass correction and finite proton size.

The experimental and the theoretical $F_{c h}(q)$, for the various cases, for the closed shell nuclei: ${ }^{4} \mathrm{He},{ }^{16} \mathrm{O}$ and ${ }^{40} \mathrm{Ca}$ are shown in Figure 1 while for the open shell nuclei: ${ }^{12} \mathrm{C},{ }^{24} \mathrm{Mg},{ }^{28} \mathrm{Si}$ and ${ }^{32} \mathrm{~S}$, are shown in Figure 2.

From the values of $\chi^{2}$, which have been found in Cases 1 and 2 (see Table I) and also from Figures 1 and 2, it can be seen that the inclusion of the correlations improves the fit of the form factor of all the nuclei we have examined. Almost all the diffraction minima which are known from the experimental data are reproduced in the correct place. There is a disagreement in the fit of the form factor of the open shell nuclei ${ }^{24} \mathrm{Mg},{ }^{28} \mathrm{Si}$ and ${ }^{32} \mathrm{~S}$ for $q \approx 3.5 \mathrm{fm}^{-1}$ where it seems that there is a third diffraction minimum in the experimental data, which cannot be reproduced in both cases.

It is seen from Table I that the parameter $b$ has the same behavior as function of the mass number $\mathrm{A}$ in the $\mathrm{HO}$ and the correlated model, while the following inequality holds:

$$
b(H O)>b(S R C) .
$$

This is due to the fact that the introduction of SRC tends to increase the relative distance of the nucleons i.e. the size of the nucleus, while the parameter $b$, which is (on the average) proportional to the (experimentally fixed) radius of the nucleus, should become smaller. Such a behavior should be expected in view also of relations (49) and (51).

It is also noted that the difference:

$$
\Delta b=b(H O)-b(S R C)
$$

is almost constant for the open shell nuclei and it is larger for the closed shell nuclei ${ }^{4} \mathrm{He},{ }^{16} \mathrm{O}$ and ${ }^{40} \mathrm{Ca}$. This can also be seen from figure 3 where the values of $\Delta b$ versus the mass number $\mathrm{A}$ has been plotted. The behavior of $\Delta b$ as function of $\mathrm{A}$ indicates that the $\mathrm{SRC}$ are stronger for the closed shell nuclei than in the open shell ones.

In Figure 4 the values of the correlation parameter $\beta$ versus the mass number $\mathrm{A}$ have been plotted. From this figure it is seen that the parameter $\beta$ is almost constant for ${ }^{4} \mathrm{He},{ }^{16} \mathrm{O}$, and ${ }^{40} \mathrm{Ca}$ and takes larger values (less correlated systems) in the open shell nuclei.

The behavior of the two parameters, $b$ and $\beta$, indicates that there should be a shell effect in the case of closed shell nuclei. That is, there is a shell effect not only on the values of the 
harmonic oscillator spacing $\hbar \omega$, as has been noted in Refs. 37, 38 but also on the values of the correlation parameter $\beta$.

In the above analysis, the nuclei ${ }^{24} \mathrm{Mg},{ }^{28} \mathrm{Si}$ and ${ }^{32} \mathrm{~S}$ were treated as $1 \mathrm{~d}$ shell nuclei. We have also considered the Case $2^{*}$ in which the occupation probability $\eta_{2 s}$ of the nuclei ${ }^{24} \mathrm{Mg},{ }^{28} \mathrm{Si}$ and ${ }^{32} \mathrm{~S}$ is taken to be a free parameter besides the other two parameters $b$ and $\beta$. We found that while the $\chi^{2}$ values become better, comparing to those of Case 2, the third diffraction minimum is not reproduced either and the behavior of the parameters $b$ and $\beta$ as functions of the mass number $A$ remains the same. The results in this case are shown in Table I and in figure 2. The values of the occupation probability $\eta_{2 s}$ of the above mentioned three nuclei are: 0.0355 , 0.0245 and 0.2945 respectively, while the corresponding values of $\eta_{1 d}$, which can be found from the values of $\eta_{2 s}$ through the relation:

$$
\eta_{1 d}=\left[(Z-8)-2 \eta_{2 s}\right] / 10
$$

are: $0.3929,0.5951$ and 0.7411 respectively. The partial occupancy of the state $2 \mathrm{~s}$ for the nucleus ${ }^{32} \mathrm{~S}$ has as result to increase the central part of the charge density significantly.

In Figures 5 and 6 the charge densities, $\rho_{c h}(r)$ (normalized to Z), of the above mentioned nuclei for the various cases of Table I are shown and compared with the charge densities of Ref. [32. In the same figures the contribution of the SRC to $\rho_{c h}(r)$,

$$
\rho_{2, c h}(r)=\rho_{c h}(r)-\rho_{1, c h}(r),
$$

is shown. The introduction of short range correlations has the feature of reducing the central part of the densities of the closed shell nuclei, while $\rho_{2, c h}(r)$ is small and characterized by oscillations in the case of open shell nuclei.

From the determined mass dependence of the parameters $b$ and $\beta$, the values of these parameters for other $\mathrm{s}-\mathrm{p}$ or $\mathrm{s}-\mathrm{d}$ shell nuclei can found. In Figures $1 \mathrm{~d}$ and $5 \mathrm{~d}$ the $F_{c h}(q)$ and $\rho_{c h}(r)$ of the nucleus ${ }^{36} \mathrm{Ar}$, treated as an $1 \mathrm{~d}$ closed shell nucleus, are shown. As there are no experimental data for $F_{c h}(q)$ for high $q$ values, the value of the parameter $\beta$ is taken to be the mean value of the corresponding values of ${ }^{16} \mathrm{O}$ and ${ }^{40} \mathrm{Ca}$, that is : $\beta_{36}=2.2937 \mathrm{fm}^{-2}$, while the parameter $b$ is determined assuming that

$$
\Delta b_{36}=\Delta b_{40}
$$

where

$$
\Delta b_{A}=b_{A}(H O)-b_{A}(S R C) .
$$

Using the values of the parameters $b_{40}(H O)=1.9453 \mathrm{fm}, b_{40}(S R C)=1.8660 \mathrm{fm}$ from Table I and choosing the parameter $b_{36}(H O)=1.8800 \mathrm{fm}$ in order to reproduce the experimental RMS charge radius of ${ }^{36} \mathrm{Ar}\left(<r^{2}>_{\text {exp }}^{1 / 2}=3.327 \pm 15 \mathrm{fm}\right.$ [32] $)$ the value $b_{36}(S R C)=1.8007 \mathrm{fm}$ was found. These values of $\beta_{36}$ and $b_{36}(S R C)$ have been used for the calculations of the correlated $F_{c h}(q)$ and $\rho_{c h}(r)$ of ${ }^{26} \mathrm{Ar}$, which are shown in figures $1 \mathrm{~d}$ and $5 \mathrm{~d}$, respectively. The calculated RMS charge radius, $\left\langle r^{2}\right\rangle^{1 / 2}=3.3343 \mathrm{fm}$, which was found, is within the experimental error.

The effect of SRC on the form factors has also been examined with various Skyrme-type wave functions. In this case, there is only one free parameter, the correlation parameter $\beta$. The potential parameters have been adjusted in Refs. [39, 40] in order to reproduce various physical quantities such as RMS radii, binding energies e.t.c.. Thus, some effects of the SRC, we would like to study, have already been averaged out. Because of that we cannot study the effect of 
the SRC on the parameters of the Skyrme interactions. This can be done if both the Skyrme parameters and the correlation parameter are readjusted by fit to various experimental data including the form factors and the fit is made for many nuclei at the same time. As this is out of the scope of the present work, we examined the form factors of ${ }^{16} \mathrm{O}$ and ${ }^{40} \mathrm{Ca}$ with various Skyrme interactions, namely SK1 to SK6 [39, 40], without SRC, named Case 3 and with SRC, named Case 4.

In Case 3 and for ${ }^{16} \mathrm{O}$, only SK1 gives smaller value for $\chi^{2}$ compared with that of Case 1 (HO without SRC). The inclusion of SRC (Case 4) to the Skyrme-type wave functions gives better $\chi^{2}$ only for the SK1, but still this value is about $10 \%$ larger than in Case 2 (HO with $\mathrm{SRC}$ ) and 1\% smaller than in Case 3. For SK2 to SK6 the correlated parameter $\beta$ goes to very large values (very small correlations) without improving the quality of the fit. For ${ }^{40} \mathrm{Ca}$ and for Case 3 all the Skyrme interactions, we have examined, give almost the same $\chi^{2}$ value which is about 20\% smaller than in Case 1 and 10\% larger than that of Case 2, while the inclusion of the correlations improves the quality of the fit, for all the Skyrme interactions, not more than $2 \%$. See Table I and figure 1 for the results we have found with SK1.

From the results with Skyrme-type wave functions we could conclude that even if a mean field is more realistic than the $\mathrm{HO}$ one, the inclusion of the SRC does not improve the fit, at least of the $F_{c h}(q)$, significantly. Significant improvement of the fit should be expected if the parameters of the mean field will be determined along with the correlation parameter. It should be noted also that, a good fit to the experimental $F_{c h}(q)$ of ${ }^{6} \mathrm{Li},{ }^{12} \mathrm{C}$ and ${ }^{16} \mathrm{O}$ has been found by Ciofi Degli Atti et al [5], using Woods-Saxon wave functions, only if the correlation parameter and the depths and radii of the wells were free parameters .

\section{SUMMARY}

In the present work, general expressions for the correlated charge form factors and densities have been found using the factor cluster expansion of Clark et al. These expressions can be used, either for analytical calculations, with HO orbitals or for numerical calculations of $F_{c h}(q)$ and $\rho_{c h}(r)$, with more realistic orbitals.

The systematic calculations, with $\mathrm{HO}$ orbitals, of these quantities, for a number of $\mathrm{N}=\mathrm{Z}$, $\mathrm{s}-\mathrm{p}$ and $\mathrm{s}-\mathrm{d}$ shell nuclei, show that there is a shell effect on the values of the HO parameter $b$ and on the correlation parameter $\beta$. Regarding the parameter $\beta$ it is almost constant for the closed shell nuclei while it takes larger values for the open shell nuclei. The mass dependence of these parameters indicates that the SRC are stronger for the closed shell nuclei than for the open shell ones. This dependence can also be used to find reasonable values of these parameters for nuclei for which there are no experimental data of the $F_{c h}(q)$.

Numerical calculations with various Skyrme-type wave functions, taken from the literature [39, 40], indicate that even if a mean field is more realistic than the $\mathrm{HO}$ one, the introduction of the SRC does not improve the fit of the charge form factors significantly. The reason for this should be that the introduction of the correlations makes a change in the mean field in a way that there is a balance between the SRC and the mean field, while for a given mean field there is not this flexibility. A way to overcome this difficulty should be to readjust the parameters of the mean field and the parameter of the correlations. 


\section{ACKNOWLEDGMENTS}

The authors would like to thank Professor M.E. Grypeos and Dr. C.P. Panos for useful comments on the manuscript.

\section{References}

[1] a. L.R.B. Elton, Nuclear Sizes (Clarendon Press, Oxford 1961).

b. H. Überall, Electron Scattering from Complex Nuclei (Academic Press, New York and London 1971).

c. R.C. Barrett, and D.F. Jackson, Nuclear Sizes and Structure (Clarendon Press, Oxford 1977).

[2] W. Czyz, and L. Lesniak, Phys. Lett. B 25, 319 (1967).

[3] F.C. Khana, Phys. Rev. Lett. 20, 871 (1968).

[4] C. Ciofi degli Atti, Nucl. Phys. A129, 350 (1969).

[5] C. Ciofi degli Atti, and N.M. Kabachnik, Phys. Rev. C 1, 809 (1970).

[6] G. Ripka, and J. Gillespie, Phys. Rev. Let. 25, 1624 (1970).

[7] M. Gaudin, J. Gillespie, and G. Ripka, Nucl. Phys. A176, 237 (1971).

[8] O. Bohigas, and S. Stringari, Phys. Lett. B 95, 9 (1980).

[9] M. Dal Ri, S. Stringari, and O. Bohigas, Nucl. Phys. A376, 81 (1982).

[10] H.P. Nassena, J. Phys. G 14, 927 (1981).

[11] a. M.V. Stoitsov, A.N. Antonov, and S.S. Dimitrova, Z. Phys. A 345, 359 (1993).

b. M.V. Stoitsov, A.N.Antonov, and S.S. Dimitrova, Phys. Rev. C 47, 2455 (1993).

[12] a. M. Grypeos, and K. Ypsilantis, J. Phys. G 15, 1397 (1989).

b. K. Ypsilantis, and M. Grypeos, 4th Hellenic Symposium on Nuclear Physics, IoanninaGreece, (1993), 99 and J. Phys. G: 21, 1701 (1995).

[13] B.A. Brown, S.E. Massen, and P.E. Hodgson, J. Phys. G 5, 1655 (1979).

[14] F. Malaguti, A. Uguzzoni, E. Verodini and P.E. Hodgson, Rivista Nuovo Cimento 5, 1 (1982).

[15] a. I.S. Gul'karov, Sov. J. Part. Nucl. 19, 149 (1988).

b. I.S. Gul'karov, and B.P. Nigam, Phys. Rev. C 52, 663 (1995).

[16] T.S. Kosmas, and J.D. Vergados, Nucl. Phys. A536, 72 (1992).

[17] S.E. Massen, H.P. Nassena, and C.P. Panos, J. Phys. G 14, 753 (1988). 
[18] S.E. Massen, and C.P. Panos , J. Phys. G 15, 311 (1989).

[19] S.E. Massen, J. Phys. G 16, 1713 (1990).

[20] R. Jastrow, Phys. Rev. 98, 1497 (1955).

[21] J.W. Clark, and M. L. Ristig, Nuov. Cim. LXXA 3, 313 (1970).

[22] M.L. Ristig, W.J. Ter Low, and J.W. Clark, Phys. Rev. C 3, 1504 (1971).

[23] J.W. Clark, Prog. Part. Nucl. Phys. 2, 89 (1979).

[24] D.M. Brink, and M.E. Grypeos, Nucl. Phys. A97, 81 (1967).

[25] H. Chandra, and G. Sauer, Phys. Rev. C 13, 245 (1976).

[26] L.J. Tassie, and F.C. Barker, Phys. Rev. 111, 940 (1958).

[27] R.R. Roy, and B.P. Nigam, Nuclear Physics, John Wiley \& Sons, Inc 1967.

[28] A. de-Shalit, and I. Talmi, Nuclear Shell Theory, Academic Press (1963).

[29] F. Arias de Saavedra, G. Co', A. Fabrocini, and S. Fantoni, Nucl. Phys. A605, 359 (1996).

[30] F. Arias de Saavedra, G. Co', and M.M. Renis Phys. Rev. C 55, 673 (1997).

[31] I.S. Gradshteyn, and I.M. Ryzhik, Tables of Integrals, Series, and Products, Academic Press (1980).

[32] H. De Vries, C.W. De Jager, and C. De Vries, Atom. Data and Nucl. Data Tables, 36, 495 (1987).

[33] a. R. Frosch, Phys. Lett. B 37, 140 (1971).

b. R.G. Arnold, B.T. Chertok, S. Rock, W.P. Schutz, Z.M Szalata, D. Day, J.S. McCarthy, F. Martin, B.A. Mecking, I. Sick, and G. Tamas, Phys. Rev. Lett. 40, 1429 (1978).

[34] I. Sick, and J.S. McCarthy, Nucl.Phys. A150, 631 (1970).

[35] B.B. Sinha, G.A. Peterson, R.R. Whitney, I. Sick, and J.S. McCarthy, Phys. Rev. C 7, 1930 (1973).

[36] G.C. Li, M.R. Yearian, and I. Sick, Phys. Rev. C 9, 1861 (1974).

[37] C.B. Daskaloyannis, M.E. Grypeos, C.G. Koutroulos, S.E. Massen, and D.S. Saloupis, Phys. Lett. B 121, 91 (1983).

[38] a. G.A. Lalazissis, and C.P. Panos, Phys. Rev. C 51, 1297 (1995).

b. G.A. Lalazissis, and C.P. Panos, Intern. Journal of Mod. Phys. E 5, 664 (1996).

[39] D. Vautherin, and D.M. Brink, Phys. Rev. C 5, 626 (1972).

[40] M. Beiner, H. Flocard, N. Van Giai, and P. Quentin, Nucl. Phys. A238, 29 (1975). 
Table 1: The values of the parameters $b$ and $\beta$, of the $\chi^{2}$, and of the RMS charge radii $\left\langle r_{c h}^{2}\right\rangle^{1 / 2}$ : contribution of the one body density (column HO), contribution of SRC (column SRC) and of the total RMS charge radii (column Total), for various $\mathrm{s}-\mathrm{p}$ and $\mathrm{s}-\mathrm{d}$ shell nuclei, determined by fit to the experimental $F_{c h}(q)$. Case 1 refers to the HO form factor, Case 2 when SRC are included. Case $2^{*}$ is the case as case 2 but with the occupation probability of the state $2 s$ taken to be a free parameter. Cases 3 and 4 refer to SK1 potential with and without SRC, respectively. The experimental RMS charge radii are from Ref. [32].

\begin{tabular}{|c|c|c|c|c|c|c|c|c|}
\hline \multirow[t]{2}{*}{ Case } & \multirow[t]{2}{*}{ Nucleus } & \multirow[t]{2}{*}{$b[\mathrm{fm}]$} & \multirow[t]{2}{*}{$\beta\left[\mathrm{fm}^{-2}\right]$} & \multirow[t]{2}{*}{$\chi^{2}$} & \multicolumn{4}{|c|}{$\left\langle r_{c h}^{2}\right\rangle^{1 / 2}[\mathrm{fm}]$} \\
\hline & & & & & $\mathrm{HO}$ & $\mathrm{SRC}$ & Total & Exper. \\
\hline 1 & ${ }^{4} \mathrm{He}$ & 1.4320 & - & 31 & 1.7651 & - & 1.7651 & $1.676(8)$ \\
\hline 2 & ${ }^{4} \mathrm{He}$ & 1.1732 & 2.3126 & 3.5 & 1.5353 & 0.5277 & 1.6234 & \\
\hline 1 & ${ }^{12} C$ & 1.6251 & - & 177 & 2.4901 & - & 2.4901 & $2.471(6)$ \\
\hline 2 & ${ }^{12} \mathrm{C}$ & 1.5923 & 3.7051 & 110 & 2.4463 & 0.2566 & 2.4597 & \\
\hline 1 & ${ }^{16} \mathrm{O}$ & 1.7610 & - & 199 & 2.7377 & - & 2.7377 & $2.730(25)$ \\
\hline 2 & ${ }^{16} \mathrm{O}$ & 1.6507 & 2.4747 & 120 & 2.5853 & 0.7070 & 2.6802 & \\
\hline 3 & ${ }^{16} \mathrm{O}$ & - & & 148 & 2.6518 & - & 2.6518 & \\
\hline 4 & ${ }^{16} \mathrm{O}$ & - & 3.0201 & 146 & 2.6518 & 0.1503 & 2.6561 & \\
\hline 1 & ${ }^{24} \mathrm{Mg}$ & 1.8495 & - & 188 & 3.1170 & - & 3.1170 & $3.075(15)$ \\
\hline 2 & ${ }^{24} \mathrm{Mg}$ & 1.8270 & 6.6112 & 161 & 3.0823 & 0.3009 & 3.0969 & \\
\hline $2^{*}$ & ${ }^{24} \mathrm{Mg}$ & 1.8315 & 7.1226 & 155 & 3.0893 & 0.2841 & 3.1023 & \\
\hline 1 & ${ }^{28} \mathrm{Si}$ & 1.8941 & - & 148 & 3.2570 & - & 3.2570 & $3.086(18)$ \\
\hline 2 & ${ }^{28} S i$ & 1.8738 & 8.2245 & 114 & 3.2249 & 0.2438 & 3.2341 & \\
\hline $2^{*}$ & ${ }^{28} \mathrm{Si}$ & 1.8743 & 8.8104 & 112 & 3.2260 & 0.2315 & 3.2343 & \\
\hline 1 & ${ }^{32} S$ & 2.0016 & - & 320 & 3.4830 & - & 3.4830 & $3.248(11)$ \\
\hline 2 & ${ }^{32} S$ & 1.9810 & 9.1356 & 270 & 3.4497 & 0.2114 & 3.4561 & \\
\hline $2^{*}$ & ${ }^{32} S$ & 1.9056 & 15.579 & 194 & 3.3284 & 0.1445 & 3.3315 & \\
\hline 1 & ${ }^{36} \mathrm{Ar}$ & 1.8800 & - & - & 3.3270 & - & 3.3270 & $3.327(15)$ \\
\hline 2 & ${ }^{36} A r$ & 1.8007 & 2.2937 & - & 3.1970 & 0.9470 & 3.3343 & \\
\hline 1 & ${ }^{40} \mathrm{Ca}$ & 1.9453 & - & 229 & 3.4668 & - & 3.4668 & $3.479(3)$ \\
\hline 2 & ${ }^{40} \mathrm{Ca}$ & 1.8660 & 2.1127 & 160 & 3.3353 & 1.1115 & 3.5156 & \\
\hline 3 & ${ }^{40} \mathrm{Ca}$ & - & & 181 & 3.4097 & - & 3.4097 & \\
\hline 4 & ${ }^{40} \mathrm{Ca}$ & - & 2.1729 & 178 & 3.4097 & 0.2349 & 3.4178 & \\
\hline
\end{tabular}



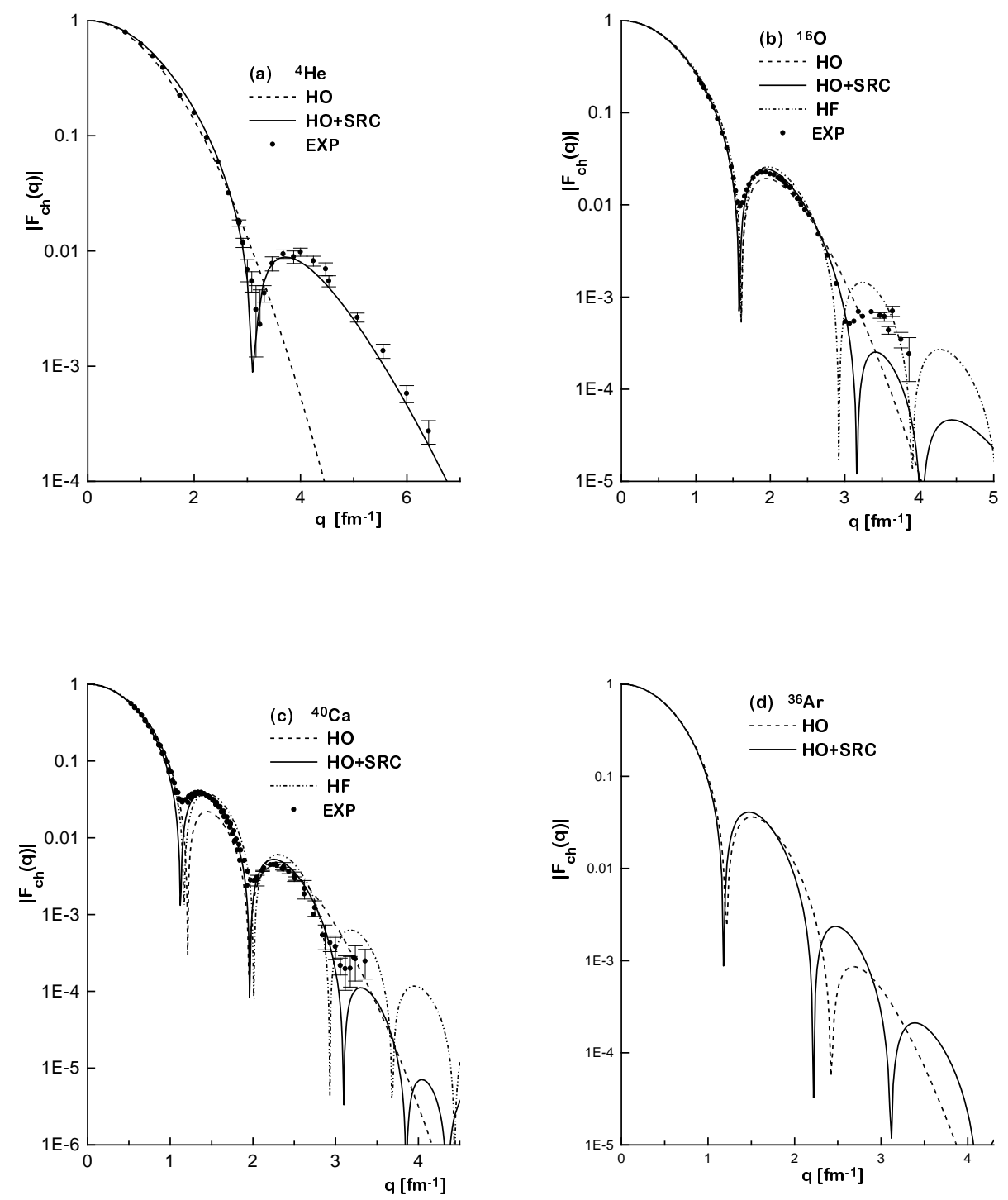

Figure 1: The charge form factor of the nuclei: ${ }^{4} \mathrm{He}(\mathrm{a}),{ }^{16} \mathrm{O}$ (b), ${ }^{40} \mathrm{Ca}$ (c) and ${ }^{36} \mathrm{Ar}(\mathrm{d})$ for various cases. The experimental points for ${ }^{4} \mathrm{He},{ }^{16} \mathrm{O}$, and ${ }^{40} \mathrm{Ca}$ are from Refs. 33, 34, 35] respectively. 

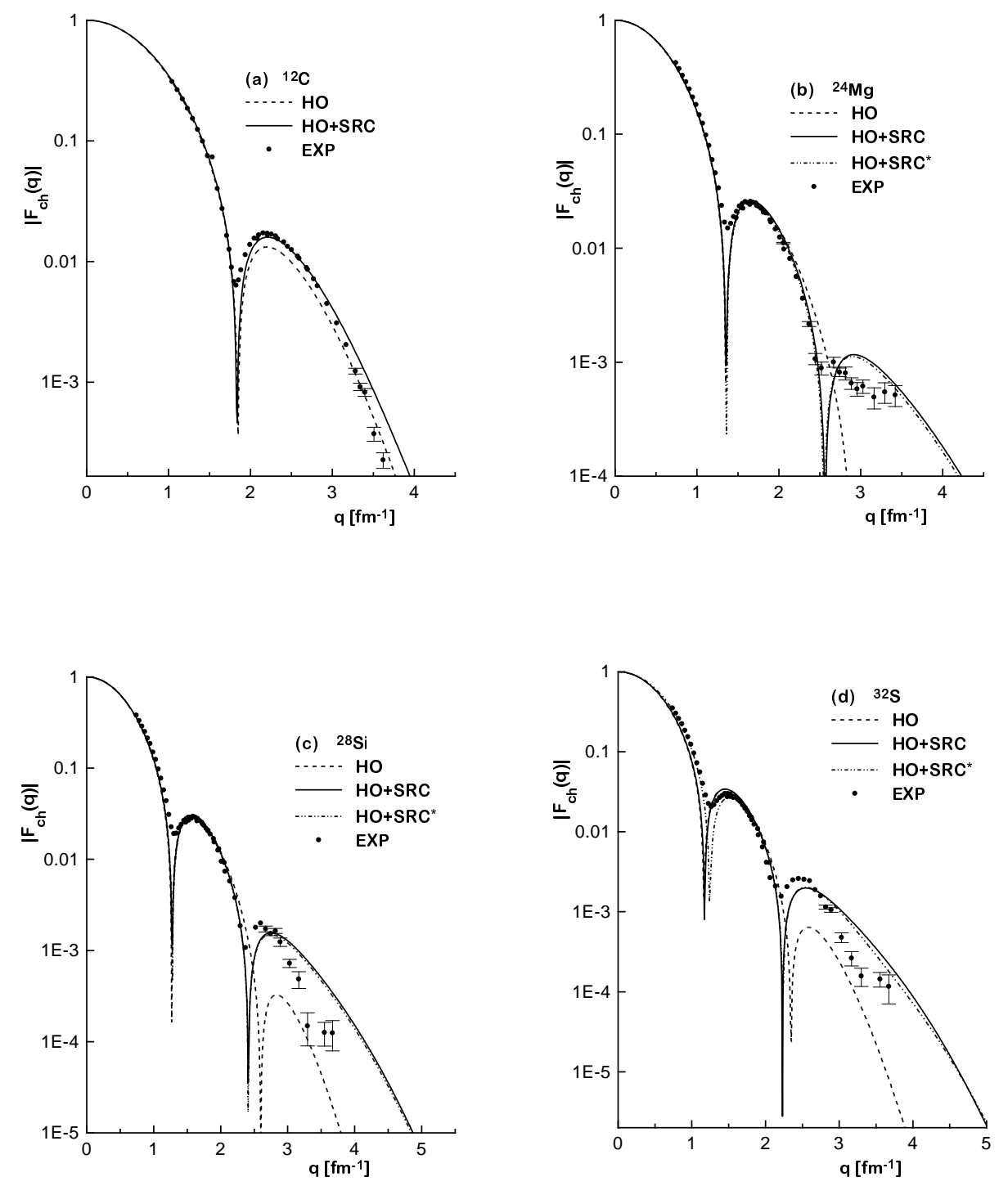

Figure 2: The charge form factor of the nuclei: ${ }^{12} \mathrm{C}(\mathrm{a}),{ }^{24} \mathrm{Mg}(\mathrm{b}),{ }^{28} \mathrm{Si}$ (c) and ${ }^{32} \mathrm{~S}$ (d) for various cases. The case $\mathrm{HO}+\mathrm{SRC}^{*}$ corresponds to the case when the occupation probability $\eta_{2 s}$ is treating as free parameter. The experimental points of ${ }^{12} \mathrm{C}$ are from Ref. [34] and for the other nuclei from Ref. [36. 


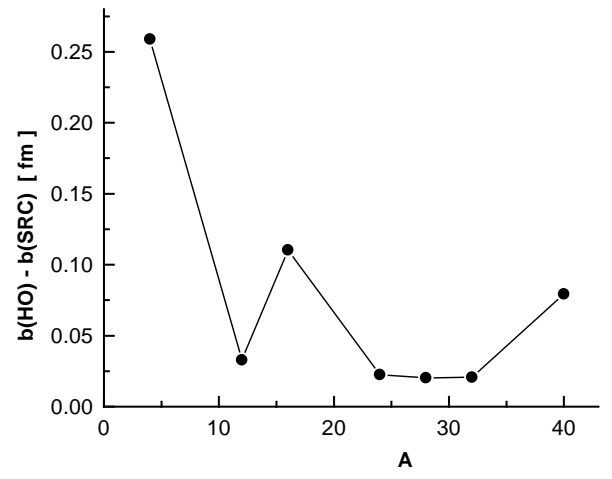

Figure 3: The difference $\Delta b=b(H O)-b(S R C)$ versus the mass number A. $b(H O)$ and $b(S R C)$ are the HO parameter in Cases 1 (HO without SRC) and 2 (HO with SRC) respectively.

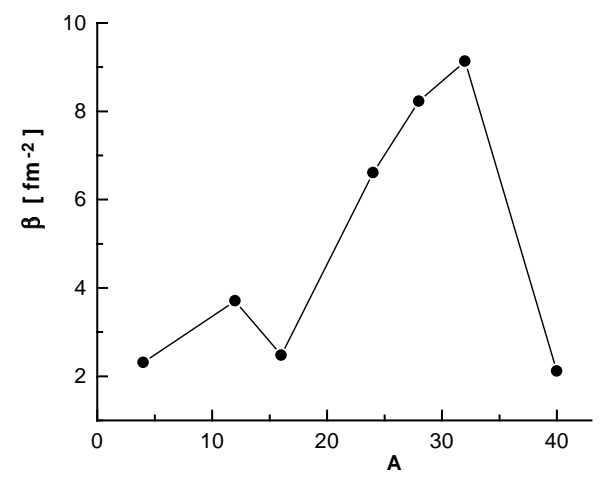

Figure 4: The correlation parameter $\beta$ versus the mass number A in Case $2(\mathrm{HO}+\mathrm{SRC})$. 

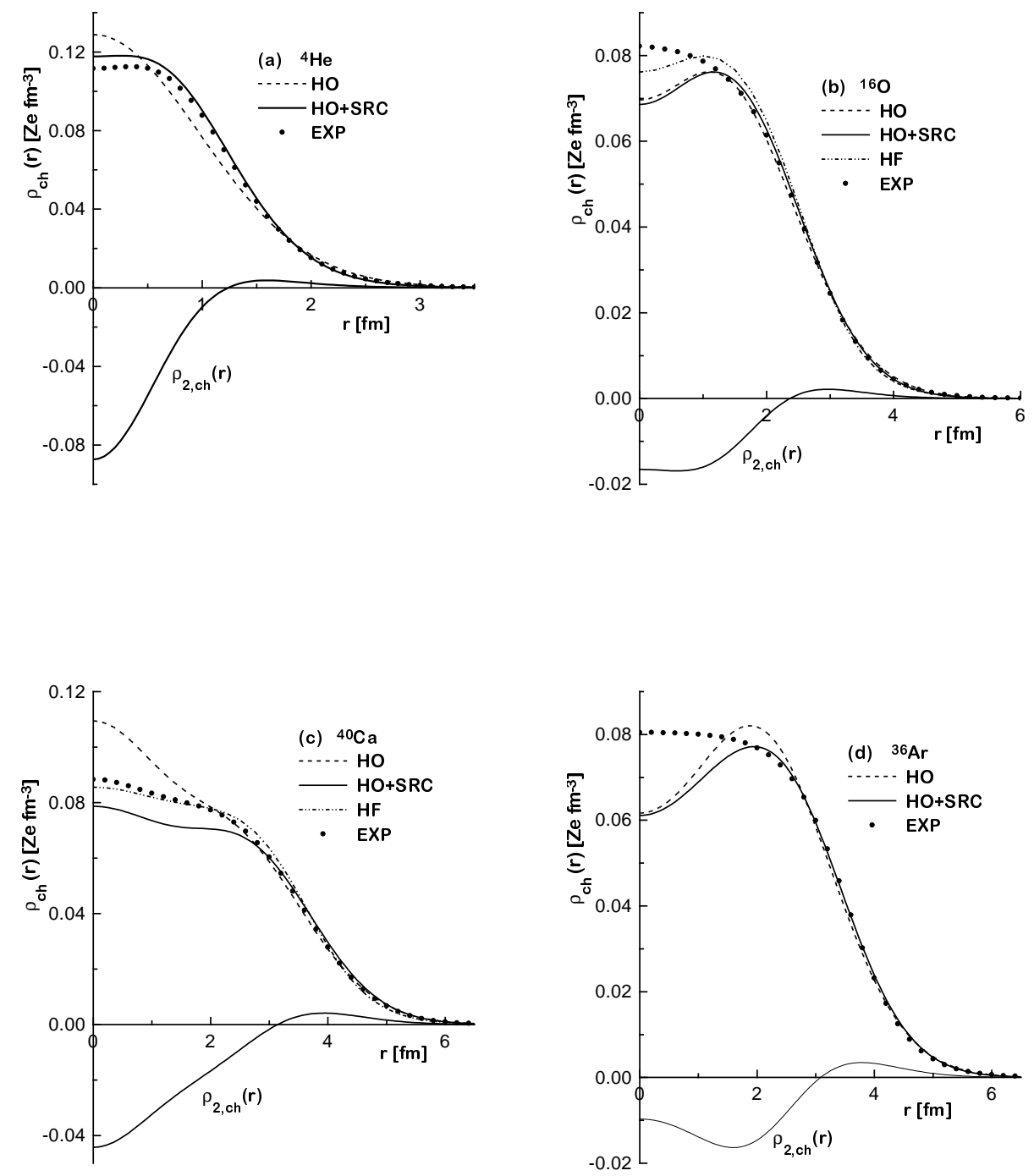

Figure 5: The charge density distribution $\rho_{c h}(r)$ and the contribution $\rho_{2, c h}(r)$ of SRC to it of the nuclei: ${ }^{4} \mathrm{He}(\mathrm{a}),{ }^{16} \mathrm{O}$ (b), ${ }^{40} \mathrm{Ca}$ (c) and ${ }^{36} \mathrm{Ar}$ (d) for various cases. The experimental points are from Ref. [32]. 

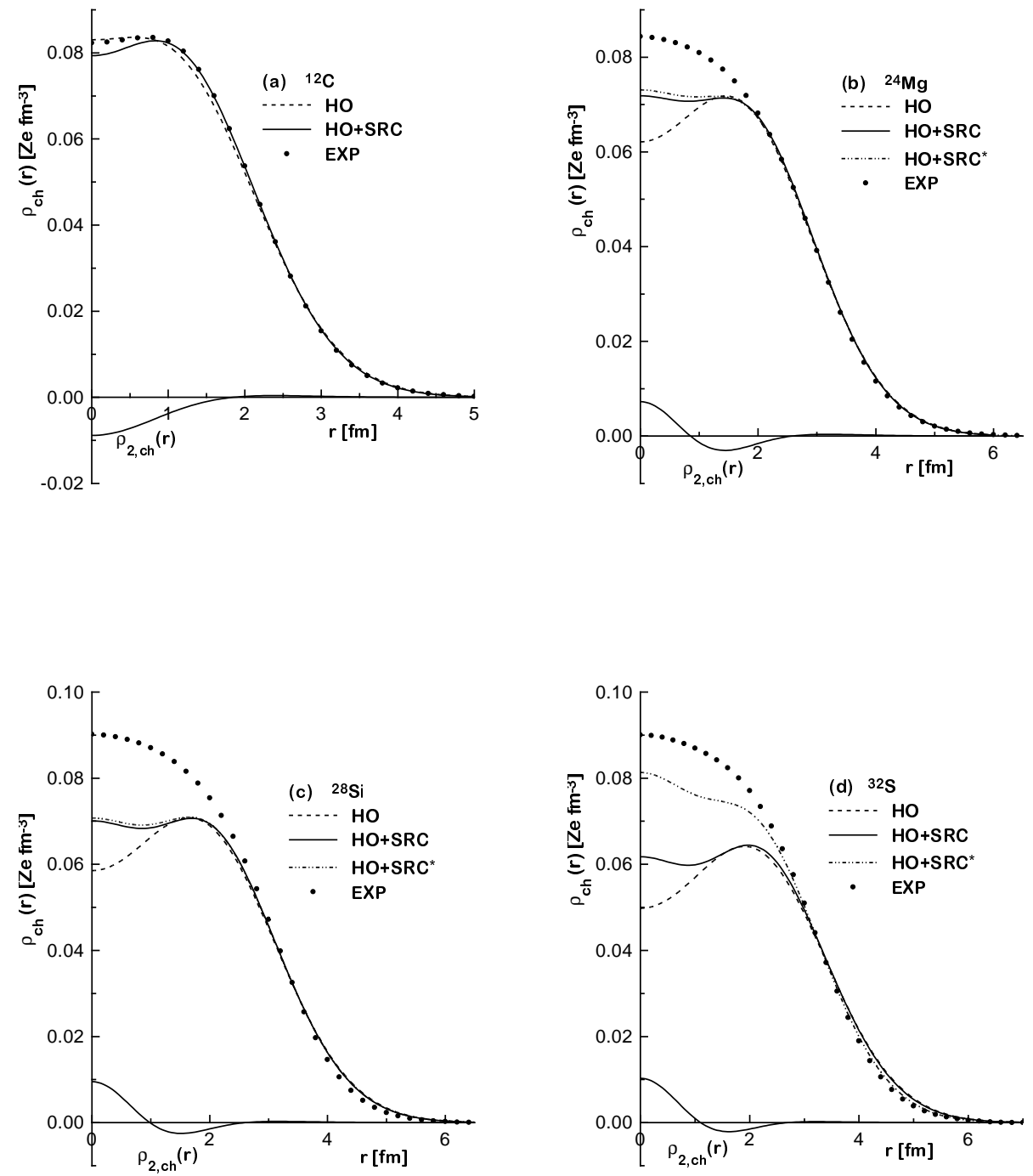

Figure 6: The charge density distribution $\rho_{c h}(r)$ and the contribution $\rho_{2, c h}(r)$ of SRC to it of the nuclei: ${ }^{12} \mathrm{C}(\mathrm{a}),{ }^{24} \mathrm{Mg}(\mathrm{b}),{ }^{28} \mathrm{Si}$ (c) and ${ }^{32} \mathrm{~S}$ (d) for various cases. The case HO+SRC corresponds to the case when the occupation probability $\eta_{2 s}$ is treated as a free parameter. The experimental points are from Ref. [32]. 